\title{
Estudo COMPARATIVO DAS COMPLICAÇÕES TERAPÊUTICAS No LÚPUS ERITEMATOSO SISTÊMICO E NAS GLOMERULOPATIAS IDIOPÁTICAS
}

\author{
*A.L. Balbi, R.A. Barbosa, M.C.P. Lima, D.B. de Almeida \\ DisciplinadeNefrologiadoDepartamentodeClínicaMédicadaFaculdadedeMedicinadeBotucatu \\ UniversidadeEstadual Paulista"JúliodeMesquitaFilho"-UNESP-Botucatu-SP
}

RESUMO - A utilização terapêutica de doses elevadas de imunossupressores pode promover diversas complicaçōes, principalmente infecciosas.

OBjetrvos. Avaliar as complicaçōes secundárias ao uso de corticóide e ciclofosfamida em portadores de nefropatias.

Métodos. Foram estudados retrospectivamente 76 pacientes atendidos no Hospital das Clínicas da Faculdade de Medicina de Botucatu - UNESP, sendo divididos em três grupos: $\mathrm{GI}=$ Lúpus Eritematoso Sistêmico sem lesão renal $(n=15) ; G 2=$ nefrite lúpica $(n=33)$ e $G 3=$ síndrome nefrótica por glomerulopatia idiopática $(n=28)$.

Resultados. Năo houve diferença em relaçäo ao tempo de acompanhamento $(G \mid=42,4 \pm 51, G 2=52,3 \pm 51, G 3=41,8 \pm$ 47,8 meses), dose total de corticóide utilizada $(G \mid=20, G 2=28$, $G 3=16$ gramas) e tempo de uso da droga $(G 1=20, G 2=26, G 3=$ 14,5 meses). Quanto ao uso de ciclofosfamida, não houve diferença na percentagem de pacientes que a utilizaram (I3\% no GI,
$51 \%$ no G2, 28\% no G3), porém pacientes do GI receberam dose total menor que G2 (mediana de zero e um grama, respectivamente $-p<0.05)$. Aspecto cushingóide, manifestaçöes gástricas, distúrbios comportamentais, diabetes mellitus e alteraçōes oculares ocorreram nos três grupos, sem diferença estatística. Quanto às complicaçöes infecciosas, aquelas consideradas clinicamente mais graves, foram mais freqüentes no $G 2(G \mid=6 \%$, $G 2=15 \%, G 3=0 \%-p<0.05)$, o mesmo ocorrendo em relação aos óbitos ( $7 \%$ no GI, 30\% no G2, 0\% no G3 - p<0.05).

Conclusōes. Pacientes portadores de nefrite lúpica apresentaram maior frequêencia de complicaçöes infecciosas decorrentes da imunossupressão prolongada, o que pode representar um marcador de gravidade deste tipo de lesão.

UnITERMOS: Lúpus eritematoso sistêmico. Nefrite lúpica. Glomerulopatiaidiopática. Imunossupressão. Complicações.

\section{INTRODUÇÃO}

Otratamento com corticóides eoutras drogas imunossupressoras éreconhecidamente acompanhado por vários tipos de complicações', principalmenteas infecciosas, sendo muitas delas potencialmente fatais. Éinegável, porém, a importância clínica desta alternativa terapêutica. Estas drogas

\footnotetext{
*Correspondência:

Departamento de Clínica Médica

Faculdade de Medicina de Botucatu - UNESP

Caixa Postal 584 - Rubião Júnior - Botucatu - SP Cep: 18618-970 - Tel.: (14) 6822-2969 Fax: (14) 6822-2238 - E-mail: abalbi@uol.com.br
}

modificaram a história natural do Lúpus EritematosoSistêmico(LES), aumentandoa expectativa devida dos pacientes ${ }^{2}$, além de atuarem em outras doenças autoimunese possibilitaremaviabilidade dos transplantes de órgãos. Amaioria dos trabalhos procura avaliar seus efeitos na recuperação ou na manutenção da função dosórgãos comprometidos, sendo os rins um dos principais alvos; porém, poucos procuram avaliar também os efeitos colaterais provocados por estasdrogas e aimportância deles na evolução da doença.

Nenhuma evidência indica que um corticóide seja mais específico que outros no tratamento do LES e de outras glomerulopatias. Geralmente, os mais usados são a hidrocortisonaea prednisona. Seus efeitos colaterais são bem estabelecidos eacometem praticamente todos os órgãos e sistemas: metabólico (obesidade central, intolerância à glicose, coma hiperosmolar não cetótico e hiperlipidemia), endócrino (supressão do eixo hipotálamo-hipófise-adrenal, redução do crescimento em criançase irregularidades menstruais), músculo-esqueléticas (osteoporose, necrose óssea de cabeça de fêmure miopatia), cutânea (fragilidade e adelgaçamento da pele, púrpura, estrias, acne ehirsutismo), ocular (cataratae 
glaucoma), sistemanervoso central (alterações psiquiátricas), cardiovasculares-renais (retenção de sódio eágua, alcalose hipocalêmica ehipertensãoarterial), gastro-intestinais (pancreatite, úlcera pépticae perfuração intestinal) e diminuição da resposta imune (infecções bacterianas, virais, fúngicas e parasitárias) ${ }^{3}$.

A ciclofosfamida é uma das drogas imunossupressoras mais utilizadas. Seus principais efeitos colaterais são bem descritos ${ }^{4}$, incluindo supressão de todos os elementos da medula óssea, cistite hemorrágica, disfunção gonadal, alopecia, teratogênese e carcinogênese ${ }^{5}$.

O acompanhamento de pacientes nefropatas quefazem uso de medicação imunossupressora em altas doses e por tempo prolongado sugere que pacientes portadores de nefrite lúpica estão mais sujeitos a complicações infecciosas quando comparados com outros pacientes portadores de diferentes patologias renais, mesmo recebendo doses semelhantes de drogas imunossupressoras, hipótese estalevantada por Staples etal. ${ }^{6}$. Em relação às complicações não infecciosas decorrentes do uso destas medicações, os dados na literatura são muito escassos, limitando-se apenas a descrever sua ocorrência, sem uma análise mais objetiva do tipo e da freqüência destas complicações.

Este trabalho tem como objetivos avaliar as complicações terapêuticas, infecciosas ou não, ocorridas em pacientes portadores de nefropatias que receberam elevadas doses de medicamentos imunossupressores e avaliar se pacientes com nefrite lúpica estão mais sujeitosa complicações infecciosas que os demais.

\section{Métodos}

Foramestudados, retrospectivamente, 76 pacientes de ambos os sexos, acima de 12 anos de idade, acompanhados pela dis- ciplina de Nefrologia da Faculdade de Medicina de Botucatu-UNESP, que fizeram uso decorticóide por tempo prolongado, sendo distribuídos em três grupos: $\mathrm{Gl}=15$ pacientes portadores de LES sem comprometimento renal; $G 2=33$ pacientes portadores de nefrite lúpica e $\mathrm{G} 3=28$ pacientes com síndromenefrótica por glomerulopatia idiopática, após descartados processos sistêmicos que possam cursar com acometimento glomerular (diabetes mellitus, doenças reumáticas, quadros infecciosos, etc).

\section{Definições:}

LES: definido deacordo com critérios da Associação Americana de Reumatologia ;

Nefrite Lúpica: presença de proteinúria (três a quatro cruzes no exame qualitativo e maior que $300 \mathrm{mg}$ em 24 horas no exame quantitativo) e/ou aumento de creatinina sérica (níveis superioresà normalidade, isto é, I,2 $\mathrm{mg} / \mathrm{dL}$ para o sexo femininoe I,5 mg/ $\mathrm{dL}$ para o sexo masculino), em pacientes portadores de LES;

- Síndrome Nefrótica:proteinúria igual ou maior que três gramas nas 24 horas;

- Pulsoterapia: administração de metilprednisolona por via endovenosa (um grama ao dia) durante três dias seguidos ou alternados;

- Complicações não infecciosas: diabetes mellitus, manifestações gástricas (epigastralgia e hemorragia digestiva alta ou baixa), osteoporose, necrose óssea de cabeça de fêmur, catarata, glaucoma, distúrbios de comportamento, aspecto cushingóide, leucopenia (leucograma mostrando menos de 4000 glóbulos brancos), neoplasias eesterilidade, clinicamente relacionadas ao uso de medicação imunossupressora. Hipertensão arterial não foi considerada como complicação secundáriaao uso do corticóide, umavez que alguns pacientes dos grupos $2 \mathrm{e} 3$ apresentavam alteração da função renal, o que poderiaalteraraavaliação desta complicação;
- Complicações infecciosas: episódios infecciosos de qualquer natureza, também clinicamente associados ao uso de imunossupressores;

- Complicações infecciosas graves: episódios infecciosos potencialmente fatais.

Quando possível, pacientes do G3 foram submetidos à biópsia renal para definição histológicadaglomerulopatia, oquenão ocorreu com os pacientes dos demais grupos.

A prednisonaea ciclofosfamidaforam as drogas de escolha, sendo calculados, para cada uma delas, dose total utilizada, dose diária média, duração total do tratamentoe, para aprednisona, o tempo total sema droga devido a interrupções por ordem médica ou abandono de tratamento. Também foram calculadas a frequiência do emprego de pulsoterapia e a média de dose de metilprednisolona utilizada, sendo a dose total incluída no cálculo da dose de corticóide recebidaem cada grupo.

Quando necessário no cálculo das doses e dos intervalos de tempo, foram feitas aproximações seguindo métodos usuais.

Para a análise estatística de dados relevantes foram empregados o teste do quiquadrado, a prova de Fruman e Halton (quando tornou-se inadequado o teste do qui-quadrado devido as baixas freqüências) e prova não-paramétrica de Kruskal-Wallis para comparação de três grupos independentes. Os valores foram considerados significativos quando $\mathrm{p}<0,05$.

\section{Resultados}

Houve predomínio do sexo feminino nos pacientes portadores de LES $(100 \%$ no GI e $91 \%$ no G2), o que não ocorreu no G3 $(50 \%)(p<0,05)$. Nãohouve diferença estatísticaem relaçãoacor, compredomínio de brancossobrenão brancos $(93 \%$ no GI, $85 \%$ no G2 e $89 \%$ no G3) (não significativo - NS). Pacientes do GI apresentaram idade superior aos do G2 (mediana de 32 e 25 
anos, respectivamente- $p<0,05)$, enquanto os pacientes do $\mathrm{G} 3$ não diferiram dos demais (mediana de 29,5 anos-NS). 71\% dos pacientes do G3 foram submetidos à biópsia renal, sendo diagnosticado glomerulonefrite membranosa (GNM) em $18 \%$, glomerulonefrite focal e segmentar (GFS) em II\%, glomerulonefrite proliferativa (GNP)em 10\% e Lesões Mínimas (LM) em $3 \%$ dos casos.

Otempodeacompanhamento médiofoi semelhante nos três grupos: $42,4 \pm 51$ meses no GI, 52,3 \pm 5 I no $G 2$ e 4I,8 \pm 47,8 no G3 (NS). Nenhum paciente foi acompanhado por um período inferioraum mês.

A tabela I mostra a utilização dos imunossupressores nos grupos estudados. Em relação à prednisona, não houve diferença estatística entre dose diáriamédia $(80 \pm 21$ $\mathrm{mg}$ no GI; $90 \pm 19$ no G2 e $87 \pm 19$ no G3 - NS), mediana de dose total utilizada $(\mathrm{GI}=20, \mathrm{G} 2=28$ e $\mathrm{G} 3=16$ gramas $\mathrm{NS})$, mediana de tempo de uso ( $\mathrm{GI}=20$, $\mathrm{G} 2=26$ e G3 = 14,5 meses $-\mathrm{NS}$ ) e média de tempo sem uso da droga $(43 \pm 76$ meses no GI; $38 \pm 46$ no $\mathrm{G} 2$ e $26 \pm 34$ no G3-NS). Nãohouve diferença estatística em relação ao emprego de pulsoterapia (I3\% dos pacientes do GI , 45\% do G2 e I I\% do G3 - NS) e na média de dose de metilprednisolona utilizada por pacienteem cada grupo $(G I=1,0 \pm 0 ; G 2=2,8 \pm 2,6$; $\mathrm{G} 3=1,0 \pm 0$ gramas $-\mathrm{NS})$. A percentagem de pacientes que receberam ciclofosfamida (I3\% no GI , 5 I\% no G2 e $28 \%$ no G3 NS) e a dose diária média ( $100 \pm 0 \mathrm{mg}$ no GI; $92,6 \pm 24,6$ no G2 e 93,7 \pm II,5 no G3-NS) também foramsemelhantes entre os grupos. Pacientes do $\mathrm{GI}$ receberam menores doses totais de ciclofosfamida quando comparados ao G2, enquanto pacientes do $G 3$ não diferiram dos demais (mediana de zero para o GI, umgrama para ○ $G 2$ e zero para ० $G 3-p<0,05)$, o mesmo ocorrendo em relação ao tempo de

Tabela I - Utilização de imunossupressores nos grupos estudados

\begin{tabular}{|c|c|c|c|c|}
\hline Imunossupressores & $\mathrm{Gl}(n=15)$ & $G 2 \quad(n=33)$ & G3 $(n=28)$ & estatística \\
\hline \multicolumn{5}{|l|}{ PREDNISONA } \\
\hline dosetotal & 20 & 28 & 16 & NS \\
\hline tempo de uso & 20 & 26 & 14.5 & NS \\
\hline tempo sem uso & $43+76$ & $38+46$ & $26+34$ & NS \\
\hline \multicolumn{5}{|c|}{ METILPREDNISOLONA } \\
\hline$\%$ uso & 13 & 45 & II & NS \\
\hline dose total & $1.0+0$ & $2.87+2.6$ & $1.0+0$ & NS \\
\hline \multicolumn{5}{|l|}{ CICLOFOSFAMIDA } \\
\hline$\%$ uso & 13 & 51 & 28 & NS \\
\hline dose diária & $100+0$ & $92+24$ & $93+11$ & NS \\
\hline dosetotal & 0 & I & 0 & $p<0.05$ \\
\hline tempo de uso & 0 & I & 0 & $p<0.05$ \\
\hline
\end{tabular}

tempo $=$ meses $;$ dose diária $=$ miligramas; dose total $=$ gramas

dose diária $=$ média $^{+}$desvio-padrão; dose total e tempo de uso = mediana

Tabela 2 - Complicaçöes não infecciosas ocorridas nos grupos estudados

\begin{tabular}{lcccc}
\hline Complicações & $\mathbf{G I}(\mathbf{n = 1 5})$ & $\mathbf{G 2}(\mathbf{n = 3 3})$ & $\mathbf{G 3}(\mathbf{n = 2 8 )}$ & estatística \\
Aspectocushingóide & $73 \%$ & $79 \%$ & $68 \%$ & NS \\
Manif. gástricas & $27 \%$ & $48 \%$ & $43 \%$ & $\mathrm{NS}$ \\
Dist. comportamento & $13 \%$ & $18 \%$ & $25 \%$ & $\mathrm{NS}$ \\
Diabetesmellitus & $7 \%$ & $3 \%$ & $7 \%$ & $\mathrm{NS}$ \\
Catarata & $7 \%$ & $3 \%$ & $4 \%$ & $\mathrm{NS}$ \\
Glaucoma & $6 \%$ & $0 \%$ & $3 \%$ & $\mathrm{NS}$ \\
Necrose óssea & $0 \%$ & $9 \%$ & $0 \%$ & $\mathrm{NS}$ \\
Osteoporose & $7 \%$ & $0 \%$ & $0 \%$ & $\mathrm{NS}$ \\
Leuopenia & $0 \%$ & $15 \%$ & $0 \%$ & $\mathrm{NS}$ \\
\hline
\end{tabular}

NS= não significante

uso (mediana de zero para o GI, um mês para o $\mathrm{G} 2$ e zero para o $\mathrm{G} 3$ - $\mathrm{p}<0,05$ ). Não houve diferença estatística entre as complicações não infecciosas ocorridas nos três grupos, conformemostradona tabela2, sendo as principais o aspecto cushingóide (73\% no GI, 79\% no G2 e 68\% no G3 $\mathrm{NS})$, manifestações gástricas $(27 \%$ no GI,
48\% no G2 e 43\% no G3-NS), distúrbios do comportamento (I3\% no GI, I8\% no G2 e $25 \%$ no G3 - NS), diabetes mellitus (7\% no GI, 3\% no G2 e 7\% no G3 - NS), catarata $7 \%$ noGI, 3\% noG 2 e $4 \%$ no G3 -NS), e glaucoma ( $6 \%$ no GI, $0 \%$ no G2e $3 \%$ no G3 -NS). Necrose óssea de cabeça defêmurocorreuno G2 ( $9 \%$ dos pacientes 
- NS) e osteoporose no GI (7\% - NS), enquanto leucopenia surgiu somenteno $\mathrm{G} 2$ (I5\% dos pacientes-NS), estando associado ao uso de ciclofosfamida.

Quanto às complicações infecciosas, o número de pacientes que apresentaram pelo menos uma infecção foi semelhante $(G I=53 \% ; G 2=73 \%$ e $G 3=43 \%-N S)$. A tabela 3 mostra as complicações infecciosas consideradas não graves ocorridas em cadagrupo. Infecções de pele (46\% no GI, $27 \%$ no G2 e $39 \%$ no G3 -NS), moniliase oral $(G \mid=13 \%, G 2=30 \%, G 3=4 \%$ NS)eotite/amigdalite bacteriana (GI $=0 \%$, $\mathrm{G} 2=12 \%, \mathrm{G} 3=0 \%$ - NS) não apresentaram diferença estatística, porém infecção urinária $(\mathrm{Gl}=20 \%, \mathrm{G} 2=51 \%, \mathrm{G3}=4 \%$ - $p<0,05)$ e infecção pulmonar ( $G \mid=7 \%$, $\mathrm{G} 2=39 \%, \mathrm{G} 3=18 \%-\mathrm{p}<0,05)$ foram mais freqüentes no $\mathrm{G} 2$ quando comparadas aos demais. Em relação às complicações infecciosas consideradas graves, mostradas na tabela 4, abortamento infectado ocorreu somente no GI (7\%), enquanto choque séptico $(6 \%)$, tuberculose de laringe (3\%), encefalite viral (3\%)e coroiditeséptica(3\%) ocorreram somente no G2. Analisadas individualmente, não houve diferença estatística entre os grupos, porém, em conjunto, as infecções graves foram mais freqüentes no $\mathrm{G} 2(15 \%)$ quando comparadas aos demais ( $G I=6 \%$ e G3 = 0\% - $p<0,05)$, conforme mostrado na figura I. Relacionando número total de infecçõesenúmerototal de pacientes em cada grupo, obtém-se índices que tambémdemonstramamaiorfreqüência decomplicações infecciosas no G2 $(0,93$ no Gl; I,76 no G2 e 0,64 no G3 - $p<0,05)$.

A figura I também mostra que a ocorrência de óbitos foi maior no G2 (30\%) quando comparadosaos demais ( $7 \%$ no GI e $0 \%$ no $G 3-p<0,05)$, sendo as causas no GI, pneumonite intersticial (100\%) e no G2, insuficiência renal (40\%), crise lúpica (20\%), insuficiência respiratória(20\%), hemorragia digestiva (10\%) e pancreatitenecro-hemorrágica( $(10 \%)$.

Tabela 3 - Complicaçōes infecciosas näo graves ocorridas nos grupos estudados

\begin{tabular}{lcccc}
\hline Complicações & $\mathbf{G I}(\mathbf{n = 1 5})$ & $\mathbf{G 2}(\mathbf{n = 3 3})$ & $\mathbf{G 3}(\mathbf{n = 2 8 )}$ & estatística \\
Infecções de pele & $46 \%$ & $27 \%$ & $39 \%$ & NS \\
Infecções urinária* & $20 \%$ & $51 \%$ & $4 \%$ & $\mathrm{p}<0.05$ \\
Infecçõespulmonar* & $7 \%$ & $39 \%$ & $18 \%$ & $\mathrm{p}<0.05$ \\
Moniliaseoral & $13 \%$ & $30 \%$ & $4 \%$ & $\mathrm{NS}$ \\
Otite/Amigdalite & $0 \%$ & $12 \%$ & $0 \%$ & $\mathrm{NS}$ \\
\hline *G2 > GI = G3 & & & & \\
\hline
\end{tabular}

Tabela 4 - Complicações infecciosas graves ocorridas nos grupos estudados

\begin{tabular}{lccccc}
\hline Complicações & $\mathbf{G I}(\mathbf{n = 1 5})$ & $\mathbf{G 2}(\mathbf{n = 3 3})$ & $\mathbf{G 3}(\mathbf{n = 2 8})$ & estatística \\
Aborto infectado & $7 \%$ & $0 \%$ & $0 \%$ & NS \\
Choque séptico & $0 \%$ & $6 \%$ & $0 \%$ & NS \\
Tuberculoselaringe & $0 \%$ & $3 \%$ & $0 \%$ & NS \\
Encefaliteviral & $0 \%$ & $3 \%$ & $0 \%$ & NS \\
Coroiditeséptica & $0 \%$ & $3 \%$ & $0 \%$ & NS \\
\hline
\end{tabular}

NS= não significante

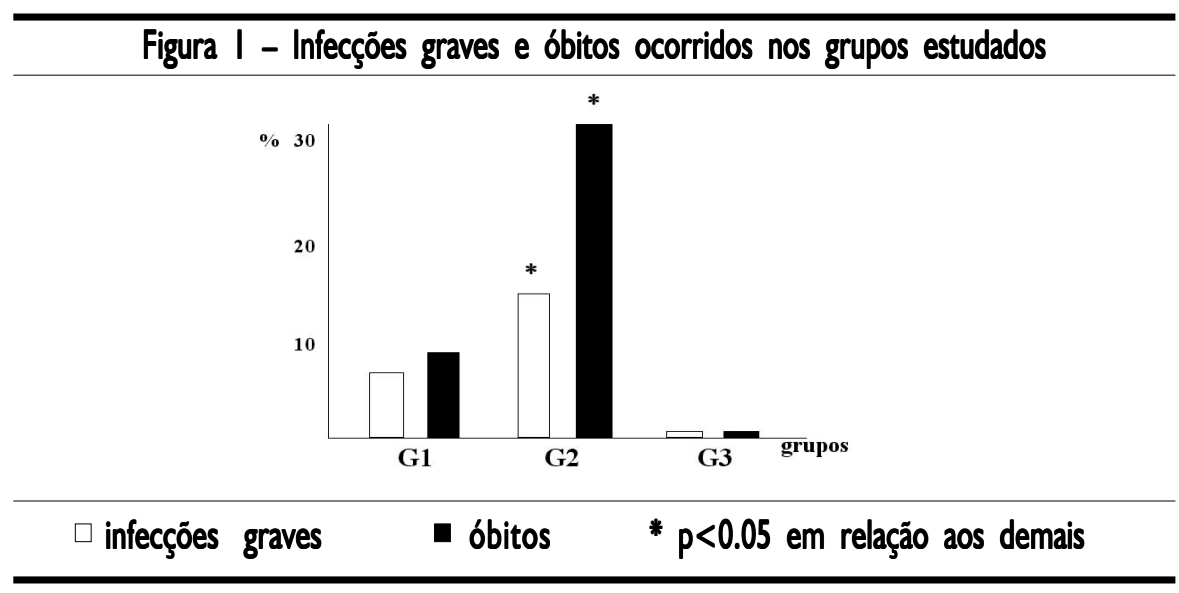

\section{Discussão}

Este estudo mostrou diversos efeitos colaterais apresentados por pacientes que fizeram uso, por tempo prolongado, de medicação imunossupressora, tanto aqueles portadores de LES, com e sem lesão renal, como também aqueles portadores deglomerulopatia idiopática.
Os grupos estudados foram semelhantes em relação a sexo, cor, tempo de acompanhamento, dose e tempo de uso de prednisona, freqüência de uso e dose total de metilprednisolona, percentagem de pacientes que receberam ciclofosfamida e dose diária média desta droga. Pacientes do $\mathrm{GI}$ apresentaram maior média de idade e menor dose total e tempo de uso de 
ciclofosfamida quando comparados aos pacientes do $\mathrm{G} 2$.

Em relação às complicações clínicas não infecciosas, casos de esterilidade, cistite hemorrágica e neoplasias, todas relacionadas ao uso de ciclofosfamida, não foram identificadas. Provavelmente, isto ocorreu devido ao curto período de uso desta droga (mediana de zero ou um mês) ou pela pequena dose total empregada (mediana de zero ou um grama). Coretteetal. ${ }^{8}$, avaliando 18 pacientes com nefrite lúpica que receberam ciclofosfamida por um período médio de 85 meses, encontraram 16\% de casos de cistite hemorrágica e $16 \%$ de neoplasias. Plotz et al. ${ }^{9}$, acompanhando 54 pacientes portadores de LESou artritereumatóideem uso de ciclofosfamida, mostraram que I $3 \%$ desenvolveram cistite hemorrágica e $4 \%$ carcinoma transicional de bexiga após 28 a 60 meses de tratamento. Leucopenia, também relacionada ao uso de cicl ofosfamida, surgiu em $15 \%$ dos pacientes do G2, com reversão após redução ou suspensão da droga, conforme relatado também por $\mathrm{Va}$ lerietal. ${ }^{10}$.

Osteoporosesurgiu emapenas 7\% dos pacientes do GI enecrose óssea de cabeça de fêmurem $9 \%$ no G2. Este fato não causa estranheza, uma vez que lesões ósseas podem ser causadas por atividade do LES, independente da corticoterapia. Naliteratura, a ocorrência de necroseóssea de cabeça de fêmur ocorre emcercade 4\%a I I\% dos pacientes que recebem corticóide e em $10 \%$ a $14 \%$ dos pacientes com LES. Zizic ${ }^{11}$ demonstrou que $52 \%$ dos pacientes lúpicos desenvolveram alguma forma de lesão óssea, sem haver diferença na dose diária de prednisona recebida em até 12 meses de acompanhamento entre os que apresentavam ou não esta lesão.

Diabetes mellitus, manifestações gástricas, distúrbios do comportamento, manifestações oculares e aspecto cushingóide surgiram de modo semelhante nos grupos estudados. Dubois etal. ${ }^{5}$, avaliando trabaIhos na literatura, mostraram que entre 207 pacientes lúpicos que receberam dose de corticóide equivalente a $100 \mathrm{mg}$ de prednisona ao dia por período prolongado, $55 \%$ apresentaram aspecto cushingóide, $34 \%$ manifestações gástricas, 3,5\% diabetes mellitus e 3,5\% alterações psiquiátricas. Segundovários autores, os quadros psiquiátricos desenvolvidos por pacientes lúpicos podem estar associados a uma psicose de base, exacerbada pelo uso do corticóide. Em relação às manifestações oculares, Williamsonetal. ${ }^{12}$ mostraram que $14 \%$ dos pacientes que receberam prednisona na dose de $15 \mathrm{mg}$ ou mais ao dia por mais de dois anos desenvolveram catarata. Bolton et al. ${ }^{13}$ mostraram que pacientes com síndromenefrótica que receberam corticóide em dias alternados por período prolongado apresentaram, como principais complicações não infecciosas, aspecto cushingóide, anemia, leucopeniae catarata.

Em relaçãoàs complicações infecciosas, o número de pacientes que apresentaram pelo menos uma infecção foi semelhante nos três grupos, variando entre $40 \%$ e $70 \%$. Infecção pulmonar e infecção urinária foram mais freqüentes no $G 2$, enquanto as demais apresentaram freqüência semelhante, embora algumas delas, pela sua importância clínica, devam ser destacadas, tais como coroidite séptica, laringite tuberculosa, encefalite viral echoque séptico, que ocorreramsomente no G2. Quando analisadas em conjunto, as infecções graves foram mais freqüentes no $\mathrm{G} 2$, o mesmo ocorrendo com oíndice obtidoatravés da relação entre número total deinfecções enúmero total de pacientes. Não foram detectadas infecções por herpes, apesar desta ser descrita como a principal infecção viral nos pacientes lúpicos. Na literatura, um dos mais completos estudos sobre infecções no LES foi feito por Ginzler etal. ${ }^{14}$, que acompanharam 223 pacientes por período médio de 35 meses, sendo diagnosticadas 163 infecções bacterianas, 195 virais ( 12 por herpes zoster) e 28 infecções oportunistas, sendo estas 40 vezes mais freqüentes nos pacientes que receberamaltas doses de corticóides, quando comparados com aqueles que não receberam. Estes autores concluíram que a nefrite lúpica ativa, a uremia e o uso do corticóideaumentama incidência de infecções. Staples et al. ${ }^{6}$ estudando 23 pacientes com LES, 20 com artrite reumatóide e II com sindrome nefrótica idiopática (a maioria recebendo doses semelhantes de corticóides), mostraram que o ritmo de infecção foi de 1.22 por 100 dias de observação para o LES e de 0.16 para os pacientes que não apresentavam LES, sendo queas infecções aumentaram na proporção da dose de corticóide utilizada e com a maior atividade lúpica. Chan etal..$^{15}$, avaliando 20 pacientes com LES que receberam prednisona e ciclofosfamida, encontraram infecção por herpes zoster ( $40 \%)$ e respiratória $(25 \%)$ como principais complicações precoces (menos de 12 meses) e tuberculose pulomonar $(25 \%)$ como principal complicação tardia (mais de 12 meses).

Amortalidade, neste trabalho, foi maior nos pacientes do $G 2$, sendo de diversas causas e podendo estar associadaàatividade da doença (criselúpica, insuficiência renal) ou ao uso do corticóide (pancreatite e hemorragia digestiva alta). Diversos trabalhos mostram que as principais causas de óbito em pacientes lúpicos são a atividade da doença eas infecções ${ }^{2,16-19}$. Appel etal. ${ }^{19}$, avaliando 150 pacientes portadores de LES, observaram mortalidade de $53 \%$, sendo insuficiência renal a causa mais freqüente. Wallace et al. ${ }^{20}$, avaliando 609 pacientes lúpicos, mostraram que aqueles com lesão renal (38\%) apresentavam menor sobrevida em semelhante tempo deacompanhamento, sendo as principais causas de óbito a insuficiência renal (32\%), sepsis (23\%), cardiovascular (I5\%) eacometimento do 
sistema nervoso central ( $10 \%)$. Rosner et al. ${ }^{17}$ mostraram que as mortes por infecção estiveram significativamente associadas a doses mais altas de corticóides, sem relação com o uso de ciclofosfamida ou de azatioprina em pacientes com LES.

Várias observações podem ser feitas a partir dos resultados obtidos neste trabalho. As complicações não infecciosas, duranteo uso de corticóde por tempo prolongado, são diversas e não parecem estar associadas ao tipo de doença, exceto lesões ósseas, especificamente relacionada ao LES. Não houve relação entre maior dose total de corticóide emaior número de complicações infecciosas, mas sim uma associação entre estasúltimas ea gravidade da doença. Como pacientes portadores de nefrite lúpica apresentarammaior freqüência de complicações infecciosas graves, pode-se considerar que esta manifestação seja um indicador de gravidade da doença. Novos estudos devem ser realizados nestesentido.

\section{AgradeCimentos}

Agradecimentos à professoraAlbina Rodrigues Torres pela revisão do texto.

\section{SUMMARY}

A comparative study OF therapeutic COMPLICATIONS IN SYSTEMIC LUPUS ERYTHEMATOSUS AND IN IDIOPATHIC GLOMERULOPATHY

PURPOSE. To evaluate the therapeutic complications due to the use of immunosupressors in patients with nephropathy.

METHODOs. 76 patients whohadusedsteroids and cyclophosphamide were retrospectivelystudied. The casesweredivided into threegroups: $G I=15$ patients with Systemic Lupus Erythematosus without renal lesion; G2=33patientswithlupusnephritisand G3= 28patients withnephroticsyndromeowingto idiopathicglomerulopathy.

RESULTS. Therewere no differences rela- ted to time of follow up $(G)=42.4 \pm 5 I$, $G 2=52.3 \pm 51, G 3=41.8 \pm 47.8$ months), total used dosage of steroids $(G 1=20, G 2=28, G 3=16$ grams) and time of drug use $(G I=20, G 2=26, G 3=$ 14.5 months). About cyclophosphamide use, there was no difference in the percentage of patients who used it (I $3 \%$ in $\mathrm{GI}$, $51 \%$ in G2, 28\% in G3), but the patients from $G$ I received lower total dosage than those from $G 2(p<0.05)$. Cushingoid appearance, epigastric distress, psychiatric disorders, diabetes mellitus and ocular alterations occurred in all the three groups, with no statistically significant differences. The infections complications, those considered more severe clinically, were more frequent in $G 2(G)=6 \%, G 2=15 \%$, $G 3=0 \%-p<0.05)$, the same occurring with the deaths (7\% in GI, 30\% in G2, $0 \%$ in $G 3-p<0.05)$.

CoNCLUSION. Inpatientswithlupusnephritis thereweremoreinfectionscomplicationsowing to prolonged immunosuppresion what may indicateaseveritymarker of thistype oflesion. [Rev Ass Med Brasil 200I; 47(4); 296-30I]

KEYWORDS: Systemic lupus erythematosus. Lupus nephritis. Idiopathicglomerulopathy. Immunossupresion. Complications.

\section{REFERÊNCIAS}

I. Meuleman J, Katz P. The immunologic effects, kinetics and use of glucocorticosteroids. Med Clin North Am 1985; 69:805-16.

2. Karsh J, Klippel JH, Balow JE, Decker JL. Mortality in lupus nephritis. Arthritis Rheum 1979; 22:764-9.

3. Nelson AM, Conn DL: Glucocorticoids in rheumatic disease. Mayo Clin Proc 1980; 55:758-69.

4. Kovarsky J. Clinical pharmacology and to xicology of cyclophosphamide: emphasis on use in rheumatic disease. Semin Arthritis Rheum 1983; 12:359-72.

5. Dubois EL, Wallace DJ. Management of discoid and systemic lupus erythematosus. In: Wallace DJ, Dubois EL, editors. Lupus erythematosus. 3rd ed. Philadelphia: Lea \& Febiger, 1987. p.50I-64.

6. Staples PJ, Gerding DN, Decker JL, Gordon RS
Jr. Incidence of infection in systemic lupus erythematosus. Arthritis Rheum 1974; 17:1-10.

7. Tan EM, Cohen AS, Fries JF, Masi AT, Mc Shane DJ, Rothifield NF, etal. The 1982 revised criteria for the classification of systemic lupus erythematosus. Arthritis Rheum 1982; 25:127I-7.

8. Corette S, Klippel JH, Decker JL, Austin HÁ, Plotz PH, Stenberg AD, et al. Controlled studies of oral immunossupressive drugs in lupus nephritis - a long-term follow-up. Ann Intern Med 1983; 99:1-8.

9. Plotz PH, Klippel JH, Decker JL, Grauman et al. Bladder complications in patients receiving cyclophosphamide for systemic lupus erythematosus or rheumatoid arthritis. Ann Intern Med 1979; 91:221-3.

I0. Valeri A, Radhakrishnan J, Estes D, D'Agati U, Kopelman R, Pernis A, et al. Intravenous pulse cyclophosphamide treatment of severe lupus nephritis: a prospective five-year study. Clin Nephrol 1994; 42:7I-8.

I I. Zizic TM, Marcoux C, Hungerford DS, Dansereau JV, Stevens MB. Corticosteroid therapy associated with ischemic necrosis of bone in systemic lupus erythematosus. Am J Med 1985; 79:596-604.

12. Williamson J, Paterson RW, McGavin DD, Jasani MK, Boyle JÁ, Doig WM, et al. Posterior subcapsular cataracts and glaucoma associated with long-term oral corticosteroid therapy in patients with rheumatoid arthritis and related conditions. Br J Ophthalmol 1969; 53:36I-72.

13. Bolton WR, Atuk NO, Sturgill BC, Westervelt FB. Therapy of the idiopathic nephrotic syndrome with alternate day steroids. Am J Med 1977; 62:60-70.

14. Ginzler E, Diamond H, Kaplan D, Weiner M, Schlesinger M, Seleznick M. Computer analysis of factors influencing frequency of infection in systemic lupus erythematosus. Arthritis Rheum 1978; 21:37-44.

15. Chan TM, LiFK, HaoWK, Chan KW, LuiSL, Tang $S$, etal. Treatment of membranous nephritis with nephrotic syndrome by se quencial immunosuppression. Lupus 1999; 8:545-5I.

16. Correia P, Cameron JS, Lian JD, Hicks J, Ogg CS, Williams DG, et al. Why do patients with lupus nephritis die? Br Med J 1985; 290: 126-3I.

17. Rosner S, Ginzler EM, Diamond HS, Weiner $\mathrm{M}$, Fries JF, Wasner C, et al. A multicenter study of outcome in systemic lupus erythematosus. II. Causes of death. Arthritis Rheum 1982; 25:612-7.

18. SteibergAD, Reinertsen JL, Plotz PH, BalowJE, Klippel JH. Systemic lupus erythematosus: envolving concepts. Ann Intern Med 1979; 91:587-604.

19. Appel GB, Silva FG, Pirani CL, Meltzer JI, Estes $D$. Renal involvement in systemic lupus erythematosus: a study of 56 patients emphasizing histologic classification. Medicine 1978; 57:37|-410.

20. Wallace DJ, Podell T, Weiner J, Klinenberg JR, Forouzesh S, Dubois EL. Systemic lupus erythematosus - survival patterns. Experience with 609 patients. JAMA 1981; 245:934-8.

Artigo recebido: 10/08/2000 Aceito para publicação: 0I/02/200 I 